\title{
AVALIAÇÃO PRELIMINAR DOS CURSOS DE GRADUAÇÃO DE UMA INSTITUIÇÃO DE ENSINO SUPERIOR PRIVADA UTILIZANDO ANÁLISE ENVOLTÓRIA DE DADOS E RESTRIÇÕES AOS PESOS
}

\author{
Darciane Alves Justino ${ }^{1}$ \\ Silvio Figueiredo Gomes Júnior ${ }^{2}$ \\ André Raeli Gomes ${ }^{3}$
}

\begin{abstract}
RESUMO
Em um mercado caracterizado por níveis cada vez maiores de competitividade e o surgimento de demandas mais complexas e em constantes mudanças para a manutenção das instituições de ensino, faz crescer a percepção de que as abordagens padrões adotadas para a avaliação de desempenho não são mais suficientes. O cenário atual requer análises multidimensionais e o referenciamento de unidades eficientes através do estabelecimento de benchmarks. Este artigo consiste em avaliar de forma quantitativa os cursos de graduação de uma Instituição de Ensino Superior (IES) Privada, localizada no interior do Estado do Rio de Janeiro, através da metodologia Análise Envoltória de Dados (DEA). Foi utilizada ainda a técnicas de restrições aos pesos para melhor avaliação destes cursos. A criação deste índice de eficiência fornece um instrumento a mais aos gestores da IES para aperfeiçoamento do processo de ensinoaprendizagem.
\end{abstract}

Palavras-chave: Análise Envoltória de Dados, Cursos de Graduação, Eficiência, Ensino Superior

\begin{abstract}
For the maintenance of educational institutions in a market characterized by high levels of increasing competitiveness and the emergence of more complex and changing demands, increases the perception that the standard approaches adopted for evaluating the performance are no longer sufficient. The current scenario requires multidimensional analysis and referencing of efficient units through the establishment of benchmarks. This article aims to assess quantitatively the undergraduate courses of a Private Higher Education Institution (IES), located in the Rio de Janeiro State using Data Envelopment Analysis (DEA) methodology. It's also used the weight restrictions techniques for better assessment of these courses. The creation of this efficiency index provides an instrument to the managers of the IES for improving the teaching-learning process.
\end{abstract}

Keywords: Data Envelopment Analysis, efficiency, highcollege, undergraduate courses.

\footnotetext{
${ }^{1}$ Faculdade Redentor, e-mail: darci_nani@ hotmail.com

${ }^{2}$ Faculdade Redentor, e-mail: silviofgj@gmail.com

${ }^{3}$ Faculdade Redentor, e-mail: araele@gmail.com
} 


\section{Introdução}

O desempenho das instituições educacionais tem sido objeto de diversas discursões por parte de profissionais da área da educação, baseando seus estudos em análises qualitativas ou quantitativas. Enfoques variados de avaliação educacional podem ser encontrados nos trabalhos de Borges e Calderón (2011), Ferreira e Tenório (2010), Caiyunet al. (2011), Warren et al. (2012), Dong e Hu (2012), Jiang (2012) e Liu e Yu (2012).

Para Soares de Mello et al. (2001), uma avaliação real da eficiência na área da educação é necessário uma metodologia que alie a subjetividade da avaliação qualitativa com a objetividade da avaliação quantitativa. Os métodos quantitativos de apoio à decisão aplicados ao setor de ensino apresentam a desvantagem de reduzida disponibilidade de dados, assim como dificuldade de incorporação de variáveis de natureza estritamente qualitativa. A abordagem da Análise por Envoltória de Dados (Data Envelopment Analysis - DEA), juntamente com outros métodos de pesquisa, vem sendo empregada na avaliação de desempenho das escolas e universidades. Belloni (2000) utilizou DEA para avaliação da eficiência produtiva de Universidades Federais Brasileiras. Outros trabalhos que utilizam esta metodologia para cálculo de eficiência ou benchmarks para professores, cursos e instituições de ensino são Angulo Mezaet al. (2012), Ahn (1987), Moita (2002), Lapa e Neiva (1996), Lapa et al. (1995, 1997), Nunes (1998), Bessentet al. (1983), Tyagiet al.(2009), Jeon e Shields (2005), entre outros.

Segundo Panepucci (2003), o aumento da utilização de DEA no setor educacional se deve ao fato deste setor ser composto por múltiplos insumos $\mathrm{e}$ múltiplos produtos, permitindo assim a utilização de DEA. Além disso, a liberdade que DEA possui para atribuir pesos às variáveis, deixa o decisor mais confortável ao utilizá-la .
Neste contexto, o objetivo deste trabalho é determinar a eficiência dos diferentes cursos de graduação de uma instituição de ensino superior privada (IES) localizada no Estado do Rio de Janeiro.

Esta IES completou 10 anos de atividades em 2012 e iniciou suas atividades com seis cursos de graduação: Engenharia Civil, Engenharia Mecânica, Nutrição, Fonoaudiologia, Ciências Biológicas e Serviço Social. Em dezembro de 2006, a faculdade passou a oferecer dois novos cursos: Fisioterapia e Sistemas de Informação. Em janeiro de 2008, a faculdade implantou mais quatro cursos: Engenharia de Produção, Administração, Enfermagem e Arquitetura e Urbanismo. Em julho de 2011, iniciou o curso de Direito.

A IES busca oferecer sempre cursos de qualidade e por isso seu crescimento acelerado vem se destacando em sua área de influência. Por esta razão, o desenvolvimento de indicadores de qualidade é fundamental para que esta instituição atinja suas metas. $E$, neste sentido, a medida de eficiência dos seus cursos de graduação com a utilização de DEA foi apoiada e encorajada pelos gestores da IES.

Para o desenvolvimento deste trabalho, foram analisados os cursos de graduação que formaram alunos no segundo semestre de 2010.

\section{Data Envelopment Analisys(DEA)}

A Análise de Envoltória de Dados (do inglês Data Envelopment Analysis) é um método não-paramétrico, surgido formalmente com o trabalho de Charnes et al. (1978), com o objetivo de medir a eficiência de unidades tomadoras de decisão, chamadas DMUs (Decision Making Units), na presença de múltiplos fatores de produção (inputs) e múltiplos produtos (outputs).

As DMU's caracterizam-se por desempenhar tarefas semelhantes, ou seja, 
utilizam os mesmos insumos e desempenham as mesmas tarefas para produzir um mesmo produto, diferindo nas quantidades de recursos (inputs) utilizados e de produtos(outputs) gerados.

A técnica de construção de fronteiras de produção e indicadores de eficiência produtiva teve origem no trabalho de Farrel (1957) e foi generalizada por Charneset al. (1978), no sentido de trabalhar com múltiplos insumos e múltiplos produtos.

\subsection{Modelos DEA Clássicos}

Há dois modelos DEA clássicos: CCR (de Charnes, Cooper e Rhodes) e BCC (de Banker, Charnes e Cooper). O modelo CCR (ou CRS - Constant Returns to Scale), trabalha com retornos constantes de escala (Charnes et al., 1978). Em sua formulação matemática considera-se que cada DMU $k, k=1, \ldots, s$, é uma unidade de produção que utiliza $n$ inputsx $x_{i k}, i=1, \ldots, n$, para produzir $m$ outputsy $_{j k}, j=1, \ldots, m$. Esse modelo maximiza o quociente entre a combinação linear dos outputs e a combinação linear dos inputs, com a restrição de que para qualquer DMU esse quociente não pode ser maior que 1 .

Mediante alguns artifícios matemáticos, este modelo pode ser linearizado, transformando-se em um Problema de Programação Linear (PPL) apresentado em (1), onde $h_{o}$ é a eficiência da DMU $o$ em análise; $x_{i o}$ e $y_{j o}$ são os inputs e outputs da $\mathrm{DMU}_{o} ; v_{i}$ e $u_{j}$ são os pesos calculados pelos modelo para inputs e outputs.

$$
\begin{aligned}
& \max h_{o}=\sum_{j=1}^{m} u_{j} y_{j o} \\
& \text { sujeito a } \sum_{i=1}^{n} v_{i} x_{i o}=1 \\
& \sum_{j=1}^{m} u_{j} y_{j k}-\sum_{i=1}^{n} v_{i} x_{i k} \leq 0, \quad k=1, \ldots, s \\
& u_{j}, v_{i} \geq 0 \quad \forall x, y
\end{aligned}
$$

ENGEVISTA, V. 16, n. 1, p. 111-121, Março 2014 


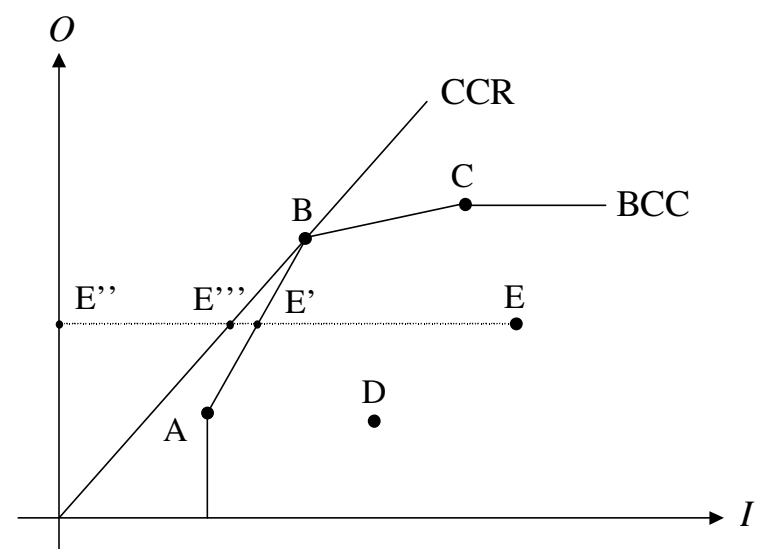

Figura 1 - Fronteiras DEA BCC e CCR para o caso bidimensional.

Fonte: Biondi Neto, 2001.

Além de identificar as DMUs eficientes, os modelos DEA permitem medir e localizar a ineficiência e estimar uma função de produção linear por partes, que fornece o benchmark para as DMUs ineficientes. Esse benchmark é determinado pela projeção das DMUs ineficientes na fronteira de eficiência. A forma como é feita esta projeção determina orientação do modelo: orientação a inputs (quando se deseja minimizar os inputs, mantendo os valores dos outputs constantes) e orientação a outputs (quando se deseja maximizar os resultados sem diminuir os recursos).

Em ambos os modelos acima, não é considerada nenhuma restrição aos pesos estipulados para os inputs e outputs, exceto serem estritamente positivos. Desta forma, o método tende a ser benevolente com as DMUs, estipulando pesos que as favoreçam. Em muitos casos, a atribuição de pesos zero empobrece a análise, pois desconsidera a variável que prejudica a eficiência de uma DMU. Uma das formas de resolver este problema é com a utilização de técnicas de restrições aos pesos.

\subsection{Restrições aos Pesos}

A incorporação de julgamento de valor através de restrições aos pesos pode ser dividida em três grupos de métodos, segundo Lins e Angulo-Meza (2000): restrições diretas nos pesos, regiões de segurança e restrições nos inputs e outpus virtuais.

\subsubsection{Restrições Diretas aos Pesos}

$\mathrm{O}$ enfoque de restrições diretas nos pesos, desenvolvido por Dyson e Thanassoulis (1988) e generalizado por Roll e Golany (1991), propõe o estabelecimento de limites numéricos aos multiplicadores, com o objetivo de não superestimar ou ignorar inputs ou outputs na análise. Este tipo de restrição pode levar à inviabilidade do PPL, uma vez que, estabelecer um limite superior ao peso de um input, implica em um limite inferior no input virtual total do resto das variáveis, e por sua vez isso tem implicações para os valores que podem tomar os inputs restantes. As equações (3) e (4) apresentam as formulações para os limites inferiores e superiores dos multiplicadores dos outputs e inputs, respectivamente.

$$
\begin{aligned}
& I I_{i} \leq v_{i} \leq S I_{i} \\
& 1 O_{i} \leq u_{i} \leq S O_{i}
\end{aligned}
$$

\subsubsection{Método de Regiões de Segurança}

O método de Regiões de Segurança (Assurance Region - AR), desenvolvido por Thompson et al. (1990), limita a variação dos pesos a uma determinada região. As restrições da abordagem por AR são de dois tipos: Tipo I (ou método Cone Rattio) e Tipo II.

Para o tipo I, é incorporada à análise a ordenação relativa ou valores relativos de inputs e outputs, as equações que representam as restrições estão apresentadas em (5) e (6).

$$
k_{i} v_{i}+k_{i+1} v_{i+1} \leq v_{i+2}
$$




$$
\alpha_{i} \leq v_{i} / v_{i+1} \leq \beta_{i}
$$

A região do segurança Tipo II, apresentada por Thomponet al. (1990) compreende restrições que relacionam os pesos dos inputs e dos outputs, conforme (7).

$$
\gamma_{i} v_{i} \geq u_{j}
$$

\subsubsection{Restrição aos Inputs e Outputs Virtuais}

Outra forma de restringir a liberdade dos pesos, conforme descrito por Branco da Silva e Soares de Mello (2005) é baseada no fato de que a contribuição de um input à DMU é $v_{i} x_{i}$. Assim, um critério de seleção pode ser o de incluir apenas os inputs e outputs que contribuem de "maneira significativa" aos custos totais e benefícios relevantes a uma DMU. Ao invés de restringir os valores dos pesos, são definidas restrições à proporção do output virtual total da DMUj, utilizado pelo output r, ou seja, a "importância relacionada" ao output $\mathrm{r}$ pela DMUj, ao intervalo $\left[\phi_{r}, \varphi_{r}\right]$, com $\phi_{r}$ e $\varphi_{r}$ sendo determinados pelo especialista (Wong e Beasley, 1990). A restrição no output $\mathrm{r}$ é apresentada em (8) onde $\sum_{r=1}^{s} u_{r} y_{r j}$ representa o output virtual total da DMUj.

$$
\varphi_{r} \leq\left(u_{r} y_{r j} / \sum_{r=1}^{s} u_{r} y_{r j}\right) \leq \phi_{r}
$$

Este artigo utiliza a técnica de região de segurança tipo I para a determinação dos pesos das variáveis e tornar mais completa a análise dos resultados. Foi escolhida a técnica de região de segurança tipo I pois esta exige menos informações por parte dos decisores. Além disso, esta técnica trabalha com informação ordinal ao invés de informação cardinal (BANA E COSTA et al, 2013). Este tipo de informação é menos precisa, gerando menos inviabilidade na determinação dos pesos das variáveis (ALLEN et al, 1997).

\section{Estudo de caso}

Foram avaliados os cursos de graduação da IES, que formaram alunos no segundo semestre de 2010. São eles: Engenharia Civil, Engenharia Mecânica, Nutrição, Fonoaudiologia, Ciências Biológicas, Serviço Social e Fisioterapia.

A escolha das variáveis do modelo tem como base os trabalhos de Senra et al. (2007), que propuseram um método para seleção de variáveis que combina a boa relação causal e boa discriminação entre as DMUs chamada de método multicritério de seleção de variáveis e Andrade et al. (2009) que utiliza este mesmo método para escolha de variáveis para avaliação de cursos de graduação do CEDERJ (Centro de Educação à Distância do Estado do Rio de Janeiro). O método de seleção de variáveis foi importante na definição da variável dos alunos ingressantes, pois, como os alunos podem ter reprovações durante o curso, o tempo de integralização do curso nem sempre é igual ao tempo que o aluno levou para concluir seus estudos. No entanto, Andrade et al. (2009) mostram que os dados relevantes são os referentes ao primeiro semestre da respectiva turma concluinte, ou seja, como estão sendo considerados os formandos do $2^{\circ}$ semestre de 2010, serão considerados os ingressantes no $1^{\circ}$ semestre de 2006 para cursos de 5 anos de duração e $1^{\circ}$ semestre de 2007 para cursos de 4 anos de duração.

Desta forma, foram consideradas 3 variáveis no modelo: 2 inputs e 1 output. Como inputs foram adotados o número de alunos ingressantes no $1^{\circ}$ semestre do curso relativo à turma que se formou $\left(1^{\circ}\right.$ semestre de 2006 para cursos de 5 anos de duração e $1^{\mathrm{o}}$ semestre de 2007 para cursos de 4 anos 
de duração) e o custo com professores no $2^{\circ}$ semestre de 2010. Cabe destacar que a utilização do custo com professores em apenas 1 semestre deve-se ao fato deste curso possuir variação muito pequena de um semestre para o outro dentro de um mesmo curso. A inclusão desta variável no modelo deve-se ao fato desta instituição ser uma instituição de ensino privada que possui recursos limitados para operar. Desta forma, o controle dos custos possui uma importante função para o sucesso da instituição. Esta foi uma alteração em relação ao trabalho de Andrade et al. (2009) que avaliarão uma instituição de ensino pública. Como output foi considerado o número de alunos concluintes no $2^{\circ}$ semestre de 2010.

A Tabela 1 apresenta a relação dos cursos e os valores dos dados normalizados de inputs e outputs utilizados no modelo, onde AI representa Alunos Ingressantes (input) e AC representa alunos concluintes (output).

Tabela 1: Dados do modelo.

\begin{tabular}{|l|c|c|c|}
\hline \multicolumn{1}{|c|}{ Cursos } & AI & Custos & AC \\
\hline Eng. Civil & 5,81 & 17,41 & 5,8 \\
\hline Eng. Mecânica & 11,62 & 13,35 & 8,38 \\
\hline Nutrição & 22,09 & 17,68 & 27,74 \\
\hline Fonoaudiologia & 6,74 & 9,58 & 7,74 \\
\hline Biologia & 14,41 & 13,97 & 9,67 \\
\hline Serviço Social & 31,62 & 13,67 & 34,83 \\
\hline Fisioterapia & 7,67 & 14,35 & 5,8 \\
\hline
\end{tabular}

O modelo DEA utilizado foi $o$ BCC, uma vez que se tem retornos variáveis de escala e a orientação utilizada foi output pois busca-se maximizar os outputs para a mesma quantidade de recursos, ou seja, o objetivo da IES é o aumento do número de alunos formados e não reduzir o número de alunos ingressantes nos cursos.

Para a resolução dos PPL's dos modelos DEA, utilizou-se o software SIAD (Sistema Integrado de Apoio à Decisão) (ANGULO MEZA et al., 2005).

\section{Análise dos Resultados}

A seguir a Tabela 2 apresenta as eficiências obtidas para cada curso avaliado.

Tabela 2: Eficiências.

\begin{tabular}{|l|c|}
\hline \multicolumn{1}{|c|}{ DMU } & Eficiência \\
\hline Engenharia Civil & 1,000000 \\
\hline Engenharia Mecânica & 0,594398 \\
\hline Nutrição & 1,000000 \\
\hline Fonoaudiologia & 1,000000 \\
\hline Biologia & 0,545296 \\
\hline Serviço Social & 1,000000 \\
\hline Fisioterapia & 0,647920 \\
\hline
\end{tabular}

Com base nos resultados apresentados na Tabela 2 observa-se que quatro curso de graduação obtiveram eficiência igual a 1 que, em DEA, são os cursos considerados eficientes: Engenharia Civil, Nutrição, Fonoaudiologia e Serviço Social.

A Tabela 3 apresenta os benchmarks para cada DMU ineficiente. Vale ressaltar que para as DMUs eficientes seus alvos são elas mesmas, por isso não aparecem na Tabela 3 .

Tabela 3: Benchmarks.

\begin{tabular}{|c|c|c|}
\hline DMU & Nutrição & Fonoaudiologia \\
\hline $\begin{array}{c}\text { Eng. } \\
\text { Mecânica }\end{array}$ & $32 \%$ & $68 \%$ \\
\hline Biologia & $50 \%$ & $50 \%$ \\
\hline Fisioterapia & $6 \%$ & $94 \%$ \\
\hline
\end{tabular}


É interessante observar que, na Tabela 3, somente os cursos de Nutrição e Fonoaudiologia foram benchmarks para os cursos ineficientes, ou seja, os cursos de Engenharia Civil e Serviço Social não são benchmarks para nenhuma outra DMU. Isto ocorre porque, apesar de serem eficientes, Engenharia Civil possui um dos maiores valores de custos com professores e o número de alunos ingressantes é muito baixo. No entanto, o modelo o torna eficiente pois consegue formar uma quantidade de alunos semelhante ao número de alunos que ingressou no curso, mesmo tendo o maior custo. No caso do curso de Serviço Social, este consegue formar a maior quantidade alunos.

A Tabela 4 apresenta os pesos atribuídos a cada variável do modelo.

Tabela 4: Pesos das variáveis.

\begin{tabular}{|l|c|c|c|}
\hline \multicolumn{1}{|c|}{ DMU } & AI & Custos & AC \\
\hline Engenharia Civil & 0,3597 & 0,0000 & 0,1724 \\
\hline Engenharia Mecânica & 0,1555 & 0,0000 & 0,1193 \\
\hline Nutrição & 0,0470 & 0,0000 & 0,0360 \\
\hline Fonoaudiologia & 0,1683 & 0,0000 & 0,1292 \\
\hline Biologia & 0,1347 & 0,0000 & 0,1034 \\
\hline Serviço Social & 0,0000 & 0,1920 & 0,0287 \\
\hline Fisioterapia & 0,2246 & 0,0000 & 0,1724 \\
\hline
\end{tabular}

Como DEA possui liberdade na escolha dos pesos das variáveis, observa-se, pela Tabela 4, que o peso da variável custo obteve peso zero em todos os cursos exceto Serviço Social, o que não é interessante para a análise, pois deseja-se considerar todas as variáveis utilizadas. No entanto, devido ao problema das multiplicidades dos pesos em DEA, é possível que existam outras combinações de pesos onde não hajam pesos nulos. Assim, esta análise não é conclusiva apesar de haver indícios de não existirem outras combinações de pesos nestes casos.

Segundo Soares de Mello et al. (2006), quando, em DEA, há preferência entre os inputs e/ou outputs, por parte dos agentes de decisão, deve-se incorporar estes julgamentos de valores aos modelos DEA por meio de restrições aos pesos, associados aos inputs e/ou outputs das unidades avaliadas.
Assim, foi utilizada a técnica de regiões de segurança (AR) tipo I de restrições aos pesos. A restrição imposta ao modelo é que o peso da variável custo dos professores $\left(\mathrm{u}_{2}\right)$ tem que ser maior ou igual ao peso atribuído à variável número de alunos ingressantes $\left(\mathrm{u}_{1}\right)$. A equação (9) apresenta a restrição adicionada ao modelo DEA.

$$
v_{2}-v_{1} \geq 0
$$

Na Tabela 5 apresenta as eficiências atribuídas a cada curso após a utilização da técnica de restrições aos pesos no modelo DEA.

Tabela 5: Eficiências com restrições aos pesos.

\begin{tabular}{|l|c|}
\hline \multicolumn{1}{|c|}{ DMU } & Eficiência \\
\hline Engenharia Civil & 0,309083 \\
\hline Engenharia Mecânica & 0,477727 \\
\hline
\end{tabular}




\begin{tabular}{|l|c|}
\hline Nutrição & 0,843121 \\
\hline Fonoaudiologia & 1,000000 \\
\hline Biologia & 0,464248 \\
\hline Serviço Social & 1,000000 \\
\hline Fisioterapia & 0,68991 \\
\hline
\end{tabular}

Analisando os resultados apresentados na Tabela 5, observa-se que apenas dois cursos são considerados eficientes: Fonoaudiologia e Serviço Social. A redução do número de DMU's eficientes é uma característica da aplicação da técnica de restrições aos pesos, pois aumenta-se as restrições do modelo

A Tabela 6 apresenta os pesos atribuídos por DEA para cada variável.

Tabela 6: Pesos das variáveis com restrições aos pesos.

\begin{tabular}{|l|c|c|c|}
\hline \multicolumn{1}{|c|}{ DMU } & Ingressantes & Custos & Concluintes \\
\hline Engenharia Civil & 4,5975 & 4,5975 & 6,0052 \\
\hline Engenharia Mecânica & 3,1821 & 3,1821 & 4,1563 \\
\hline Nutrição & 0,9613 & 0,9613 & 1,2556 \\
\hline Fonoaudiologia & 3,4452 & 3,4452 & 3,4452 \\
\hline Biologia & 2,7576 & 2,7576 & 3,6019 \\
\hline Serviço Social & 0,0000 & 0,0000 & 1,0000 \\
\hline Fisioterapia & 4,5975 & 4,5975 & 6,0052 \\
\hline
\end{tabular}

Observa-se pela Tabela 6 que, com a utilização da restrição aos pesos, houve redução da quantidade de pesos nulos (com valor zero). Além disso, o modelo conseguiu encontrar solução eficiente atribuindo pesos iguais para as variáveis de input. Esta característica foi considerada positiva pelos autores pois não se sentiam confortáveis para definir qual variável era mais importante.

A Tabela 7 apresenta os Benchmarks para cada curso avaliado.

Tabela 7: Benchmarks utilizando restrições aos pesos.

\begin{tabular}{|l|c|c|}
\hline \multicolumn{1}{|c|}{ DMU } & Fonoaudiologia & Serviço Social \\
\hline Engenharia Civil & $59 \%$ & $41 \%$ \\
\hline Engenharia Mecânica & $64 \%$ & $36 \%$ \\
\hline Nutrição & $7 \%$ & $93 \%$ \\
\hline Biologia & $52 \%$ & $48 \%$ \\
\hline Fisioterapia & $71 \%$ & $29 \%$ \\
\hline
\end{tabular}

Observa-se que o fato dos cursos de Nutrição e Engenharia Civil possuírem os maiores custos (Tabela 1) faz com que deixem de serem eficientes quando se utiliza a técnica de restrição aos pesos pois, desta forma, o peso atribuído a esta variável deixa de ser nulo.

Outro ponto interessante é o curso de Nutrição possui como benchmark 
principal o curso de Serviço Social que possui a quantidade de alunos ingressantes e alunos concluintes aproximados, diferenciando apenas na variável custo dos professores.

Vale ressaltar ainda a baixa eficiência do curso de Engenharia Civil com a utilização da técnica de restrições aos pesos. Isto ocorre porque o curso de Engenharia Civil possui o segundo maior custo com professores e um número baixo de alunos ingressantes e alunos concluintes.

\section{Considerações Finais}

A técnica DEA verifica se cada unidade opera de maneira eficiente ou não, relativamente a um conjunto específico de recursos utilizados e de resultados obtidos, fazendo uma comparação entre unidades que realizam tarefas similares, sem a necessidade de conhecermos qualquer relação de importância (pesos) entre as variáveis consideradas. Desta forma, DEA se mostrou bastante apropriado para avaliação dos cursos de graduação da IES analisada. Além disso, DEA auxilia na definição em estratégias de produção que maximizem a eficiência das DMUs avaliadas, corrigindo as ineficientes através da determinação de alvos, o que pode ser bastante útil para melhoria dos cursos estudados.

Vale ressaltar que este estudo é inovador na busca da eficiência dos cursos de graduação da IES em questão, permitindo assim uma visão geral destes cursos de graduação, comparados entre si.

Como trabalhos futuros, pretende-se testar outras técnicas para maior descriminação dos mod elos DEA como, por exemplo, a inclusão de DMUs artificiais ao invés da técnica de restrições aos pesos. Deseja-se também ampliar o estudo com a inclusão de novas variáveis consideradas importantes ao estudo, definidas em acordo com os gestores da Instituição, além da inclusão de dados de outros semestres, assim como dos demais cursos que ainda não possuem alunos formados.

\section{Agradecimentos}

A FAPERJ e a IES pelo apoio financeiro.

\section{Referências Bibliográficas}

AHN, T. S. (1987) Efficiency and related issues in higuer education: a Data Envelopment Analysis approach. Ph.D. Dissertation, The Universityof Texas at Austin.

ALLEN, R.; ATHANASSOPOULOS, A.; DYSON, R.G. e THANASSOULIS, E. (1997)Weightsrestrictionsandvaluejudgeme nts in data envelopment analysis: evolution, developmentand future directions. AnnalsofOperationsResearch, vol. 73, 13-34.

ANDRADE, F. V. S.; BRANDÃO, L. C. e SOARES de MELLO, J. C. C. B. (2009) Avaliação de um curso de matemática à distância com modelos DEA e seleção de variáveis. Relatórios de Pesquisa em Engenharia de Produção da UFF, vol. 9, n. 10.

ANGULO MEZA, L.; BIONDI Neto, L.; SOARES de MELLO, J. C. C. B. e GOMES, E. G. (2005) ISYDS - Integrated System for Decision Support (SIAD Sistema Integrado de Apoio a Decisão): a software package for data envelopment analysis model. Pesquisa Operacional, vol. 25, n. 3, 493-503.

ANGULO MEZA, L.; SOARES de MELLO, J. C. C. B. e GOMES JÚNIOR, S. F. (2012) Benchmarking Distance Learning Centers with a Multiobjective Data Envelopment Analysis Model. Advanced Topics in Applied Operations Management.ed. InTech, 183-200.

BANA e COSTA, C. A.; 
ANGULOMEZA, L. e OLIVEIRA, M. D. (2013) O método MacBeth e Aplicação no Brasil. Engevista, vol. 15, n. 1. 3-27.

BANKER, R. D.; CHARNES, A. e COOPER, W. W. (1984) Some models for estimating technical scale inefficiencies in data envelopment analysis. Management Science, vol. 30, n. 9, 1078-1092.

BELLONI, I. (2000) Uma Metodologia de Avaliação da Eficiência Produtiva de Universidades Federais Brasileiras. Tese de Doutorado, UFSC.

BESSENT, A. M.; BESSENT, E. W.; CHARNES, A.; COOPER, W. W.; Thorogood, N. C. (1983) Evaluation of Educational-Program Proposals by Means of DEA. Educational Administration Quarterly, vol. 19, n. 2, 82-107.

BIONDI NETO, L. (2001) Neuro-DEA Nova Metodologia para Determinação da Eficiência Relativa de Unidades Tomadoras de Decisão. Tese de Doutorado, Universidade Federal do Rio de Janeiro.

BRANCO da SILVA, B. P. e SOARES de Mello, J. C. C. B. (2005) Modelo DEA Aplicado aos Resultados das Olimpíadas de Atenas 2004. Anais do VIII SPOLM Simpósio de Pesquisa Operacional e Logística da Marinha, Rio de Janeiro.

BORGES, R. M. e CALDERÓN, A. I. (2011) Avaliação educacional: O estado do conhecimento da revista ensaio: Avaliação e políticas públicas em educação (19932008). Ensaio, vol. 19, n. 70, 42-56.

CAIYUN, S.; YICHEN, W. e JIANJUN, Z. (2011) Model research on teaching evaluation for university teachers based on developmental evaluation. Lecture Notes in Electrical Engineering, vol. 112, 149-154.

CHARNES, A.; COOPER, W. W. e RHODES, E. (1978) Measuring the efficiency of decision-making units. European Journal of Operational Research, vol. 2, 429-444.
DONG, S. S. e HU, Y. N. (2012) The application effectiveness evaluation of the educational equipment based on AHP. Advanced Materials Research, vol. 341342, 384-389.

DYSON, R. G. e THANASSOULIS E. (1988) Reducing weight flexibility in DEA. Journal of the Operational Research Society, vol. 39, 563-576.

FARRELL, M. J. (1957) The Measurement of Productive Efficiency. J.R. Statisstic. Soc. A120, 253-290.

FERREIRA, R. A. e TENÓRIO, R. M. (2010) A construção de indicadores de qualidade no campo da avaliação educacional: Um enfoque epistemológico. RevistaLusofona de Educação, vol. 15, 7197.

JEON, Y.; SHIELDS, M. P. (2005) Integration and utilization of public education resources in remote and homogenous areas: A case study of the upper peninsula of Michigan. Contemporary Economic Policy,vol. 23,n. 4, 601-614.

JIANG, Q. Q. (2012) Teaching quality evaluation based on covariance analysis method. Lecture Notes in Electrical Engineering, vol. 100, 1032-1037.

LAPA, J. S.; LOPES, A. L. M. e LANZER, E. (1995) Análise Envoltória de Dados aplicada à avaliação de IES: determinação dos pesos relativos e valoração dos insumos e produtos. XXVII SBPO Simpósio Brasileiro de Pesquisa Operacional, Vitória.

LAPA, J. S.; BELLONI, J. A. e NEIVA, C. C. (1997) Medidas de desempenho de universidades acadêmicas de uma Instituição de Ensino Superior. Relatório Técnico- Departamento de Engenharia de Produção e Sistemas, UFSC.

LAPA, J. S. e NEIVA, C. C. (1996) Avaliação em educação: comentários sobre desempenho e qualidade. Ensaio, vol. 4, n. $12,213-236$ 
LINS, M. P. E. e ANGULO MEZA, L. (2000). Análise Envoltória de Dados e perspectivas de integração no ambiente de Apoio à Decisão. Editora da COPPE/UFRJ, Rio de Janeiro.

LIU, Y. e YU, J. (2012) A qualitative analysis and statistics of educational evaluation results based on decision tree algorithm. AdvancedMaterialsResearch, vol. 433-440, 319-323.

MOITA, M. H. V. (2002) Um Modelo para Avaliação da Eficiência Técnica de Professores Universitários utilizando Análise de Envoltória de Dados. Dissertação de Mestrado em Engenharia de Produção, Universidade Federal de Santa Catarina, Florianópolis.

NUNES, N. (1998) Avaliação da eficiência produtiva de departamentos universitários: uma aplicação de Análise Envoltória de Dados. Dissertação de Mestrado, UFSC.

PANEPUCCI, G. T. M. (2003) Avaliação de desempenho dos departamentos acadêmicos da UFSCar utilizando AnáliseEnvoltória de Dados - DEA. Dissertação de Mestrado em Engenharia de Produção, Universidade Federal de São Carlos, São Carlos.

ROLL, Y. e GOLANY, B. (1991) Controlling factor weights in DEA. IIE Transactions, vol. 23, n. 1, 2-9.

SENRA, L. F. A. C.; NANCI, L. C.; SOARES de MELLO, J. C. C. B. e ANGULO MEZA, L. (2007) Estudo sobre métodos de seleção de variáveis em DEA. Pesquisa Operacional, vol. 27, n. 2, 191207.
SOARES de MELLO, J. C. C. B.; LETA, F. R.; FERNANDES, A. J. S.; VAZ, M. R.; SOARES DE MELLO, M. H. C. e BARBEJAT, M. E. R. P. (2001) Avaliação qualitativa e quantitativa: uma metodologia de integração. Ensaio. Avaliação e Políticas Públicas em Educação, vol. 9, 237-251.

SOARES DE MELLO, J. C. C. B.; GOMES, E. G.; ANGULO MEZA, L.; SOARES DE MELLO, M. H. C. E SOARES DE MELLO, A. J. R. (2006) Engineering post-graduate programmes: a quality and productivity analysis. Studies in Educational Evaluation, vol. 32, n. 2, 136-152.

THOMPSON, R. G.; LANGEMEIER, L. N.; LEE, C. H.; LEE, E. e THRALL, R.M. (1990) The role of multiplier bounds in efficiency analysis with application to. Kansas Farming. Journal of Econometrics, vol. 46, 93-108.

TYAGI, P.; YADAV, S. P.; SINGH, S. P. (2009) Relative performance of academic departments using DEA with sensitivity analysis.Evaluation and Program Planning.Vol. 32,n. 2, 168-177.

WARREN, S. J.; DONDLINGER, M. J.; MCLEOD, J. e BIGENHO, C. (2012) Opening the Door: An evaluation of the efficacy of a problem-based learning game. Computers and Education, vol. 58, n. 1, 397-412.

WONG, Y. e BEASLEY, J. (1990) Restricting Weight Flexibility in DEA. Journal of Operational Research Society, vol. $41,829-835$. 